\title{
RELACIONES SUELO-PLANTA EN BOSQUES DE ABIES PINSAPO BOISS. DISPONIBILIDAD DE NUTRIENTES Y ESTATUS NUTRICIONAL
}

\author{
José LIÉTOR, Juan Carlos LINARES, Juan Manuel MARTÍN GARCÍA, \\ Roberto GARCÍA RUIZ, y José Antonio CARREIRA
}

\begin{abstract}
RESUMEN. Relaciones Suelo-Planta en Bosques de Abies pinsapo Boiss. Disponibilidad de Nutrientes y Estatus Nutricional. Se han evaluado las relaciones entre las propiedades del suelo y el estado nutricional de los árboles en pinsapares del Paraje Natural Los Reales de Sierra Bermeja y del Parque Natural Sierra de las Nieves (Málaga-España). Se seleccionaron pinsapares que difieren en cuanto a su estado sucesional (masas agradativas versus maduras) y sustrato litológico (serpentinas versus calizas), en los que se evaluaron variables morfoedáficas de perfiles de suelo, y se analizaron las concentraciones de nutrientes en muestras de suelo, hojarasca y acículas. Los suelos de pinsapares calcáreos en fase agradativa (Yunquera) mostraron las menores concentraciones de macro y micronutrientes totales. Esto se correspondía con contenidos también mínimos de $\mathrm{N}$ y $\mathrm{P}$ en tejidos foliares, indicando la existencia de un estrés nutricional general, como es habitual en masas forestales en fase de exclusión de tallos (máxima competencia intraespecífica). No obstante, la presencia de relaciones N/P foliares normales implica que dicho estrés nutricional no ha desencadenado desbalances internos entre dichos nutrientes en los árboles. En pinsapares calcícolas maduros (Ronda), la mayor acumulación de materia orgánica en la hojarasca y el suelo superficial se relaciona con un aumento de la disponibilidad de nutrientes en el suelo, y una reducción en el estrés nutricional de los árboles. El pinsapar serpentinícola de Los Reales de Sierra Bermeja mostró niveles anormalmente elevados de N, y de las relaciones N/P, tanto en el suelo como en los tejidos foliares. Estos síntomas son característicos de ecosistemas forestales en una fase temprana del denominado síndrome de saturación de nitrógeno, asociado a disfunciones en el ciclo del $\mathrm{N}$.
\end{abstract}

Palabras clave. Abies pinsapo, Sierra de las Nieves, Sierra Bermeja, macronutrientes, relaciones sueloplanta.

ABSTRACT. Soil-Plant relationships in Abies pinsapo Boiss-Fir Forests. Nutrient Availability and Nutritional Status. Relationships between soil profile properties and the tree nutritional status have been evaluated in Pinsapo-fir (Abies pinsapo, Boiss.) forests at Los Reales de Sierra Bermeja Natural Site and Sierra de las Nieves Natural Park (Málaga-Spain). Pinsapo stands widely differing in sucesional status (agradative versus old-growth stands) and lithology (serpentinic versus calcareous substrate) were selected. Soil profile-development and nutrients in soil, littler and needles have been compared to the plant community development and parent material. Overall macro- and micronutrients availability was low in young calcareous pinsapo stands (agradative phase). Foliar tissues at these stands showed minima $\mathrm{N}$ and $\mathrm{P}$ concentrations, indicating a general nutritional stress; although nutritional unbalances were absence as indicated by normal N/P ratios in foliar tissues. As a consequence of the progressive build-up of the organic matter pool in the litter and surface soil layers along with succession, nutrient availability in calcareous pinsapo forests was the highest at the old-growth stands, and the tree nutritional stress appeared to be relieved. The serpentinic pinsapo forest at Los Reales de Sierra Bermeja showed very high levels of nitrogen and N/P ratios, both in the soil and foliar tissues. These preliminary results suggest this pinsapo forest is at an early phase of the $\mathrm{N}$ saturation syndrome.

Keywords. Abies pinsapo, Sierra de las Nieves, Sierra Bermeja, macronutrients, plant-soil relations. 


\section{INTRODUCCIÓN}

Los pinsapares quizás sean la formación boscosa más original de la Península Ibérica. En primer lugar, debido al carácter endémico de la especie arbórea dominante (Abies pinsapo, Boiss.), abeto de indudable valor biogeográfico y paisajístico (Ruiz, 1994). Además, la extensión de los pinsapares es muy reducida, limitándose su área de distribución básicamente a tres núcleos del extremo occidental de la cordillera Bética: la Sierra de las Nieves y Sierra Bermeja en la provincia de Málaga, y la Sierra del Pinar en la de Cádiz. Por otra parte, la localización de sus masas, restringidas a montañas de clima mediterráneo pero con elevadas precipitaciones, junto a su fisonomía más propia de bosques de climas templados, o su composición florística, son otras tantas características que justifican el estudio y la conservación del pinsapar.

En Andalucía, actualmente, la mayor parte de los pinsapares se encuentran protegidos por la Ley 2/1989, de 18 de Julio, por la que se aprueba el Inventario de los Espacios Naturales de Andalucía y se establecen medidas adicionales para su protección; en concreto como Parques Naturales "Sierra de Grazalema" (1984) y "Sierra de las Nieves" (1989) y como Paraje Natural "Los Reales de Sierra Bermeja" (1989). Los dos primeros han sido además declarados como Reservas de la Biosfera por la UNESCO en 1977 y 1995 respectivamente.

En el ámbito europeo, los pinsapares quedan incluidos en la Directiva 92/43, relativa a la conservación de los Hábitats Naturales y de la Fauna y Flora Silvestres, como tipo de hábitat natural para cuya conservación es necesario designar zonas especiales de conservación. Igualmente aparecen recogidos en la transposición de esta Directiva a España; por el Real Decreto $1997 / 1995$ por el que se establecen medidas para contribuir a garantizar la Biodiversidad mediante la conservación de los Hábitats Naturales y de la Fauna y la Flora
Silvestres. Como especie, el pinsapo se encuentra protegido por el Decreto 104/94 que establece el Catálogo Andaluz de Especies de la Flora Silvestre Amenazada.

A pesar de su singularidad sólo existen algunos estudios edáficos, mayoritariamente de carácter descriptivo y centrados en el pinsapar de la Sierra de Grazalema (Corral et al., 1980; Paneque y Corral, 1982; González, 1983; Mérida, 1983; Benítez, 1984; Del Toro et al., 1996). Los trabajos edafológicos realizados en los pinsapares de la provincia de Málaga han estado orientados principalmente al conocimiento de los procesos de alteración geoquímica y de las peculiares propiedades químicas de los suelos desarrollados sobre serpentinas y peridotitas en Los Reales de Sierra Bermeja (Yusta, 1984). Sin embargo, aunque los principales problemas que hoy afectan a la gestión y conservación de los pinsapares (síntomas de declive, afección por parásitos, etc.) probablemente estén relacionados con el estatus nutricional de los árboles (estrés, deficiencias, etc.), son inéditos los estudios que aborden las relaciones suelo-planta en los pinsapares desde una perspectiva ecológicofuncional.

Las aproximaciones utilizadas tradicionalmente para evaluar la fertilidad del suelo y su aptitud para garantizar el desarrollo de determinadas comunidades vegetales han sido diversas, no existiendo un indicador universal de fertilidad del suelo. Por esta razón, las aproximaciones basadas en la calidad de la materia orgánica y la disponibilidad de nutrientes asimilables contemplan diferentes parámetros edáficos al mismo tiempo (Killham, 1994). En este sentido, las variables referidas al subsistema suelo superficial-hojarasca resultan las más adecuadas para el caso de los ecosistemas forestales. Dicho compartimento concentra los mayores contenidos de nutrientes esenciales en formas biodisponibles para las plantas, las mayores cantidades de raíces finas, las actividades microbianas más intensas y los 
procesos biogeoquímicos más activos (Waring y Schlesinger, 1985; Paul y Clark, 1989; Van Miegroet et al., 1990).

Por otra parte, la mayoría de las demandas de nutrientes en plantas se dirigen al follaje (Ranger et al. 1997). Así, en distintas especies arbóreas se ha mostrado cómo la infertilidad del suelo se manifiesta en una reducción de las concentraciones foliares de nutrientes y/o del tamaño de las hojas, y en síntomas de declive (Grubb 1998). El análisis foliar presenta algunas limitaciones para evaluar estrés nutricional debido a que el contenido foliar en nutrientes varia a lo largo del año y dentro de cada árbol cambia con la edad de la hoja, su posición en el dosel, etc. No obstante constituye una herramienta adecuada como indicador del estatus nutricional, si sus resultados se interpretan en términos relativos a través del cálculo de relaciones nutriente / nutriente (Van den Driessche 1974, Robert et al. 1996).

\section{OBJETIVOS}

Estudiar la influencia de la litología y del estado sucesional forestal sobre las relaciones suelo-planta y sus consecuencias ecológicofuncionales en pinsapares. Dicho objetivo es abordado integrando la información procedente de tres enfoques: i) el estudio edafológico clásico de perfiles de suelo, ii) la medida de distintas fracciones de $\mathrm{N}$ y $\mathrm{P}$ en el horizonte superficial y la hojarasca, y iii) los contenidos foliares de estos elementos.

\section{MATERIAL Y MÉTODOS}

\section{Área de estudio}

Nuestro estudio se centra en las masas de pinsapar del Parque Natural Sierra de las Nieves y del Paraje Natural Los Reales de Sierra Bermeja (Málaga). La Sierra de las Nieves es la parte más elevada de la Serranía de Ronda.
Se asienta sobre material calizo y margocalizo parcialmente metamorfizado de edad jurásica, y dolomías localmente metamorfizadas del triásico. En ella aparecen zonas donde se han producido fuertes karstificaciones debido a la conjunción de elevadas precipitaciones con el substrato.

Sierra Bermeja constituye uno de los mayores afloramientos del mundo de una roca intrusiva de carácter ultrabásico, donde predominan peridotitas ricas en minerales pesados. El resto de características del medio físico son similares para todas las parcelas seleccionadas (tab. 1): altitud entre 1200 y $1360 \mathrm{~m}$, relieve escarpado, orientación septentrional y estacionalidad mediterránea. La precipitación anual supera los $1000 \mathrm{~mm}$ (1135 mm en Sierra de las Nieves y $1915 \mathrm{~mm}$ en Sierra Bermeja durante el periodo 19982001 -Liétor 2001-, la mayor parte de la cuál se concentra entre Octubre y Mayo).

El pinsapar de la Sierra de las Nieves se clasifica dentro de la serie basófila mesosupramediterránea, húmeda-hiperhúmeda del pinsapo, paeonio broteroi-Abieteto pinsapi $S$. El bosque de Los Reales de Sierra Bermeja pertenece a la serie serpentinícola, mesosupramediterránea, húmeda del pinsapo, Bunio macucae-Abieteto pinsapi S. (Pérez la Torre et al, 1998).

\section{Métodos de muestreo, descripción de perfiles y análisis de laboratorio.}

Para la selección de las parcelas de estudio se llevó a cabo una prospección inicial empleando fotografías aéreas y mapas topográficos y geológicos. Se seleccionaron 4 parcelas sobre calizas de $0.5-1$ ha. de superficie en la serranía de Ronda, representativas de la variabilidad sucesional existente. El contraste entre distintas litologías se efectuó comparando una de estas parcelas con otra, sucesionalmente similar pero localizada en Los Reales de Sierra Bermeja, sobre serpentinas.

En cada una de las parcelas se efectuó una 


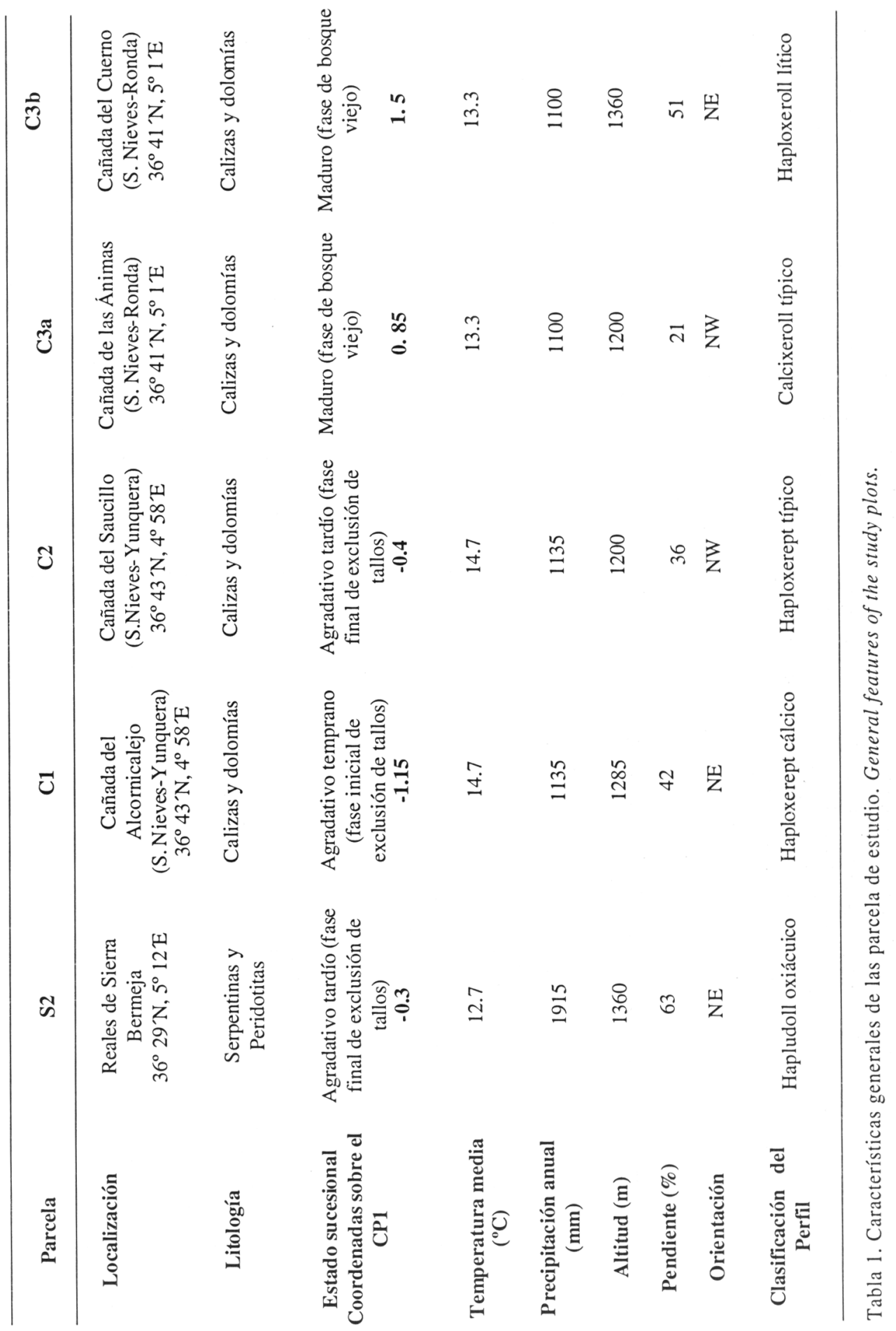


calicata en el suelo. La descripción macromorfológica de los perfiles se realizó según FAO (1977), Soil Survey Staff (1993) y Munsell Color Co. (1990), y se clasificaron con el sistema Soil Taxonomy (SSS, 1999). Para cada horizonte se analizaron las siguientes propiedades (Soil Conservation Service, 1972; Comisión de Métodos Analíticos del Instituto Nacional de Edafología, 1984): Granulometría de las fracciones arcilla $(0-2 \mu \mathrm{m})$, limo $(2-$ $50 \mu \mathrm{m})$, arena $(50-2000 \mu \mathrm{m})$ y fragmentos gruesos (>2000 $\mu \mathrm{m}$ ) (pipeta de Robinson y tamizado); $\mathrm{pH}$ en una suspensión tierra fina:agua destilada 1:1 (peso:peso); Carbono orgánico por oxidación con dicromato potásico; Nitrógeno total por digestión con ácido sulfúrico y sulfato de plata; Carbonato cálcico equivalente por volumetría de gases; Conductividad eléctrica del extracto de saturación; Capacidad de retención de agua de la tierra fina a 33 y $1500 \mathrm{kPa}$ (membrana de presión de Richards); Bases de cambio y capacidad de cambio catiónico por desplazamiento con acetato amónico $(\mathrm{pH} 7)$ y cloruro sódico.

Se estimaron elementos extraíbles ( $\mathrm{Ca}$, $\mathrm{Mg}, \mathrm{Na}, \mathrm{K}, \mathrm{Mn}, \mathrm{Fe}$ y $\mathrm{Cu}$ ) con DTPA (Lindsay y Novell, 1978). Na y K se determinaron por fotometría de llama, y Ca, Mg, Mn, Fe y Cu por absorción atómica.

Para el estudio del suelo superficial se tomó suelo de los 10 primeros centímetros del horizonte superficial con un cilindro de $6 \mathrm{~cm}$ de diámetro. El muestreo se realizó seleccionando en cada parcela 10 puntos al azar. En cada uno de ellos se obtuvo una muestra compuesta a partir de 3 submuestras. Se calculó la densidad aparente de la tierra fina, la densidad de la grava y la capacidad de campo. Para los análisis químicos de la hojarasca, se tomaron 10 muestras por parcela (capas $\mathrm{L}+\mathrm{F}$ ) utilizando un marco de muestreo de 40 x 40 $\mathrm{cm}$. La cantidad de hojarasca por unidad de superficie en cada parcela se midió a partir de 10 muestras (capas $\mathrm{L}+\mathrm{F}+\mathrm{H}$ ) de $0.16 \mathrm{~m}^{2}$ distribuidas al azar. Estas muestras se limpiaron de piedras y se tamizaron a $1 \mathrm{~mm}$. La fracción de $<1 \mathrm{~mm}$ se estimó de acuerdo con el método propuesto por Herrick (1995).

Tanto en las muestras de suelo como en las de hojarasca se analizaron: $\mathrm{C}$ y $\mathrm{N}$ totales (Autoanalizador Elemental Leco CHNS-932); formas de $\mathrm{N}$ inorgánico $\left(\mathrm{N}_{\mathrm{i}}\right)$ (amonio y nitrato), extraíbles con $\mathrm{KCl} 2 M$ y determinadas colorimétricamente (método del azul indofenol para amonio y método de Griess-Ilosvay por conversión a nitrito en columna reductora de cadmio para nitrato) (Keeney y Nelson, 1982); y $\mathrm{P}$ inorgánico $\left(\mathrm{P}_{\mathrm{i}}\right)$, extraído con $\mathrm{KCl} 2 \mathrm{M}$ en la hojarasca y con $\mathrm{NaHCO}_{3} 0.5 \mathrm{M}$ en el horizonte superficial y determinado por colorimetría (John, 1970).

Para las acículas se seleccionaron en cada parcela 4 árboles al azar, en los cuales se recolectaron acículas de 1 año de edad. En el pinsapo, esta diferenciación es sencilla gracias a la generación anual de tramos independientes en el follaje. Las muestras se congelaron hasta su análisis. Se determinaron los contenidos de $\mathrm{N}$ y $\mathrm{P}$ totales mediante digestión con ácido sulfúrico y peróxido de hidrógeno (Parkinson \& Allen 1975 ) y el C total (autoanalizador elemental Leco CHNS-932).

Como variable global cuantitativa indicadora del estado sucesional de las diferentes masas de pinsapar incluidas en este estudio, se emplearon las coordenadas de cada parcela sobre el primer eje generado por un Análisis de Componentes Principales (ACP) efectuado a partir de 7 variables estructurales medidas en las distintas parcelas (coeficiente de variación ( $\mathrm{CV}$ ) de las densidades de individuos en las distintas clases de diámetro, número de clases de diámetro, porcentaje de claros, área basal total cubierta por tallos de pinsapo, número de árboles vivos con $h>4 \mathrm{~m}$, rango diametral de los árboles vivos ( $\varnothing$ máximo- $\varnothing$ mínimo del tronco en cada parcela) y los valores de importancia de las plántulas) (Liétor 2001). Esta ordenación ha 
sido contrastada con el grado de evolución edáfica y los niveles de nutrientes en suelo, hojarasca y acículas.

\section{Análisis estadístico.}

La existencia de diferencias estadísticamente significativas, tanto entre pinsapares agradativos litológicamente contrastados como entre pinsapares calcáreos sucesionalmente contrastados, fue comprobada mediante un diseño de ANOVA simple, seguido del Test de Tukey HSD.

\section{RESULTADOS Y DISCUSIÓN}

Los resultados y su discusión aparecen divididos en dos apartados. En el primero se comparan los diferentes estadios sucesionales de los pinsapares calcáreos de la serranía de Ronda atendiendo a las relaciones suelo-planta. El segundo apartado muestra los datos obtenidos en la parcela sobre serpentinas (Sierra Bermeja), y los compara con los mostrados por la parcela, sucesionalmente similar, sobre calizas. Las figuras y tablas presentan de forma conjunta los resultados de todas las parcelas, si bien, los referidos a la parcela S2 no son abordados hasta llegar al segundo bloque.

\section{Cambios sucesionales en las propiedades edáficas de pinsapares calcícolas. \\ 1.1 Perfiles y suelo superficial.}

Los suelos de pinsapares jóvenes corresponden a inceptisoles con un horizonte orgánico poco potente; Haploxerept cálcico en el caso de la parcela Cl (pinsapar en fase inicial de exclusión de tallos), y Haploxerept típico en C2 (pinsapar en fase final de exclusión de tallos). Estos suelos contrastan con los Mollisoles que aparecen en los pinsapares maduros: Calcixeroll típico en la parcela C3a y Haploxeroll lítico en $\mathrm{C} 3 \mathrm{~b}$, cuyos porcentajes de carbono orgánico y $\mathrm{N}$ superficiales son dos
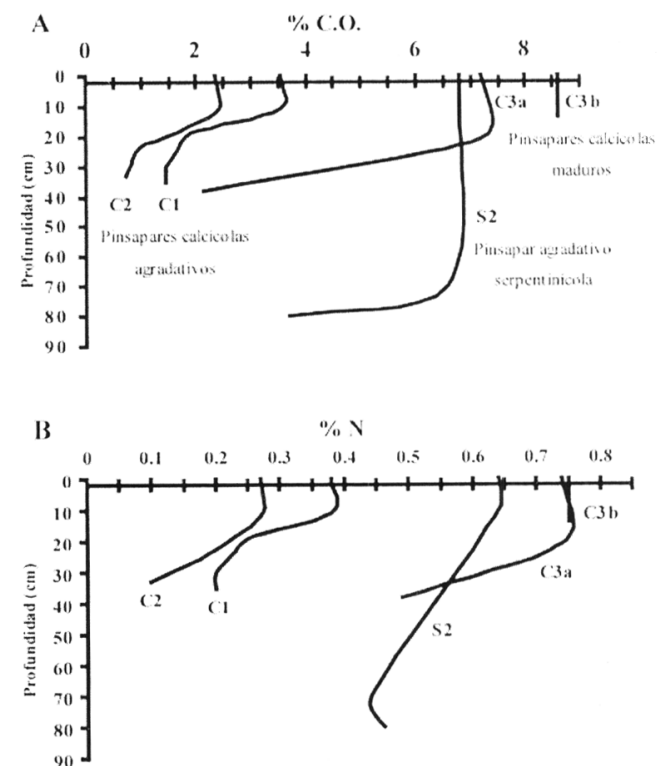

Figura 1. Distribución en el perfil de las concentraciones de Carbono Orgánico (A) y de Nitrógeno Total (B) a lo largo de una serie sucesional de pinsapares calcícolas que incluye masas en fase agradativa (Sierra de las Nieves-Yunquera, parcelas $\mathrm{C} 1$ y $\mathrm{C} 2$ ) y en fase madura (Sierra de las NievesRonda, parcelas C3a y $\mathrm{C} 3 \mathrm{~b}$ ); y en un pinsapar serpentinícola en fase agradativa (Reales de Sierra Bermeja, parcela S2 cuya estructura del dosel es comparable al de la parcela C2). Soil profile distribution of the Organic Carbon (A) and Total Nitrogen $(B)$ concentrations along a calcareous, pinsapo-firforest, sucesional serie including stands in agradative (Sierra de las Nieves, plots $C l$ and C2) and old-growth (Sierra de las Nieves, Plots $C 3 a$ and $C 3 b)$ phases; and in a serpentinic, agradative, pinsapo-fir forest (Reales de Sierra Bermeja, plot 52 which canopy structure is comparable to that in the $C 2$ plot).

veces superiores (fig. 1).

En este sentido, desde el punto de vista de la clasificación de los suelos, hay una clara correspondencia entre el grado de madurez de la comunidad vegetal y el desarrollo edáfico. Sin embargo, la secuencia de horizontes (sequum) de los pinsapares agradativos (Ah$\mathrm{B}(\mathrm{w})-\mathrm{C})$ revela un nivel de estructuración mayor al de los bosques más maduros (Ah-R). En este sentido, algunos autores han criticado 


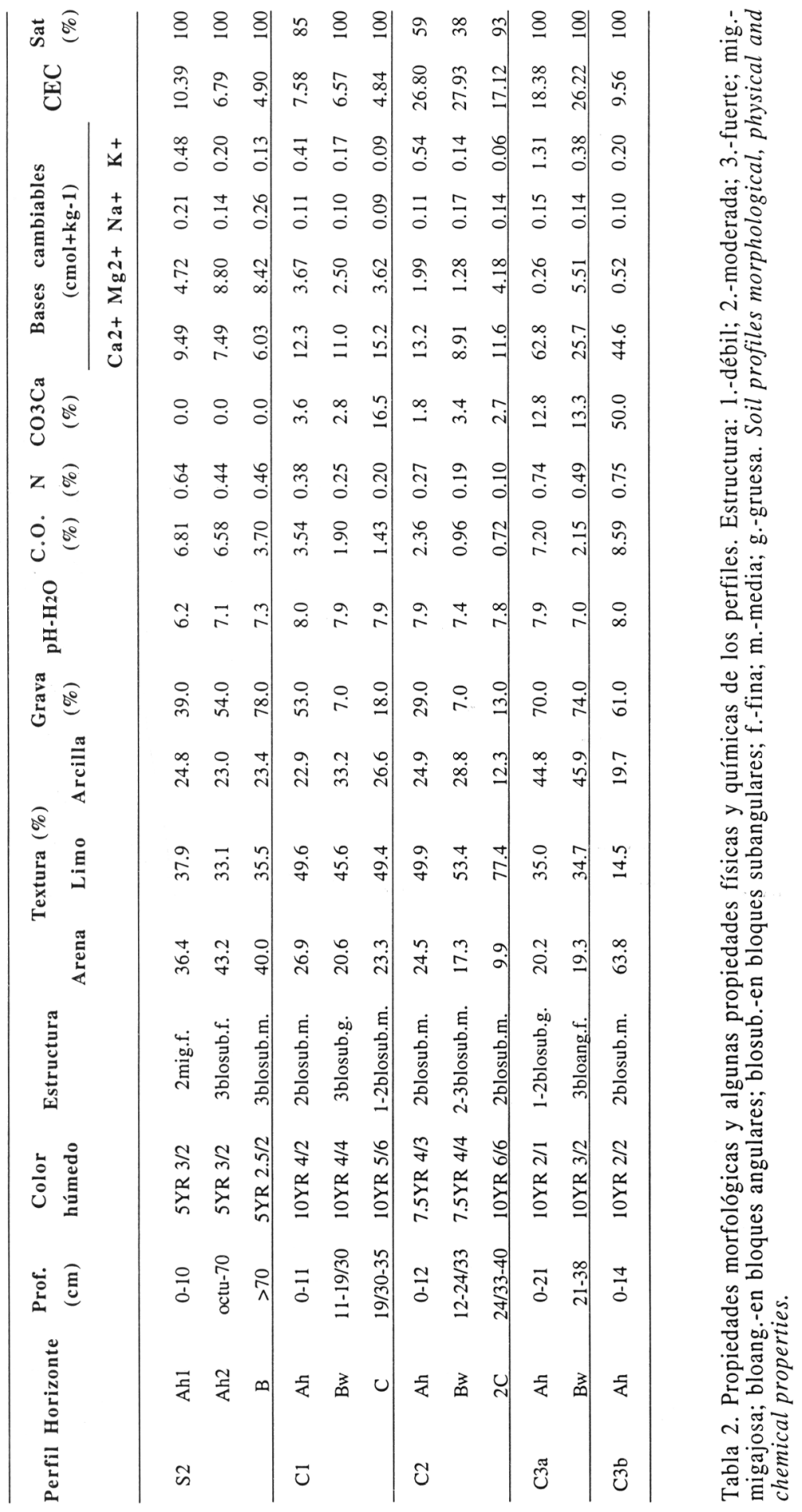


que Soil Taxonomy da más importancia a la presencia o ausencia de un horizonte de diagnóstico que al perfil en si mismo (Muir, 1969). En los pinsapares agradativos el sequum es típico de suelos mediterráneos poco evolucionados, con epipedion óchrico. Dichos pinsapares son relativamente jóvenes $(<60$ años) y se asientan en laderas que fueron objeto de cultivos marginales en el pasado. El horizonte superficial es relativamente pobre en materia orgánica, de poco espesor y estructura moderada. Los pinsapares maduros presentan un horizonte de diagnóstico móllico, con buena estructura y alto contenido en materia orgánica, asentado directamente sobre la roca madre. La estructura es característica de suelos forestales de zonas de montaña (rendsinas) (tab. 2).

El coeficiente de correlación del contenido total de $\mathrm{C}$ del suelo fue de -0.98 con la densidad aparente y de 0.99 con la capacidad de campo $(\mathrm{P}<0.01, \mathrm{n}=6)$. En este sentido, la acumulación en el suelo de restos orgánicos a lo largo de la sucesión permite mantener niveles elevados de humedad durante periodos más prolongados (Liétor 2001). Esto es de especial relevancia en ecosistemas mediterráneos de áreas montañosas, sometidos a fuertes tasas de erosión por escorrentía superficial y donde el estrés hídrico estival es fuertemente limitante (Carreira, J.A et al, 1996).

La progresiva capitalización de M.O. constituye un incremento de las reservas de macro y micronutrientes. Los pinsapares calcáreos en fase agradativa presentan los contenidos totales más bajos, apreciándose en la figura 1 que tanto para el C.O. como para el $\mathrm{N}$ quedan diferenciados de los pinsapares maduros. Lo mismo sucede al comparar los niveles de micronutrientes y oligoelementos asimilables (tab. 3). La figura 2 muestra asimismo el incremento sucesional en los contenidos de $\mathrm{Ca}^{2+} \mathrm{y} \mathrm{Mg}^{2+}$ asimilables, aunque el cociente entre ambos permanece aproximadamente constante.

\subsection{Hojarasca}

El accidentado relieve de los pinsapares, caracterizado por fuertes pendientes y abundantes afloramientos rocosos, hace que la capa de hojarasca se distribuya de forma muy heterogénea. Por esta razón, aunque existe una tendencia al incremento de la cantidad de hojarasca por unidad de superficie a lo largo de

\begin{tabular}{ccccccccc}
\hline Perfil & Horizonte & Ca & Mg & Na & K & Mn & Fe & Cu \\
\hline \multirow{5}{*}{ S2 } & Ah1 & 4.27 & 44.72 & 3.12 & 2.16 & 0.57 & 17.60 & 0.21 \\
& Ah2 & 4.60 & 62.02 & 1.64 & 0.88 & 5.15 & 17.60 & 0.21 \\
& B & 4.00 & 62.56 & 1.92 & 0.56 & 3.06 & 13.20 & 0.21 \\
C1 & Ah & 25.41 & 6.90 & 0.62 & 1.64 & 9.68 & 13.20 & 0.22 \\
& Bw & 29.52 & 6.95 & 0.88 & 0.76 & 0.24 & 5.28 & 0.18 \\
& C & 27.35 & 16.07 & 0.86 & 0.44 & 5.28 & 5.28 & 0.21 \\
C2 & Ah & 19.60 & 2.71 & 0.88 & 2.00 & 6.60 & 26.40 & 0.18 \\
& Bw & 30.73 & 2.90 & 2.16 & 0.60 & 4.40 & 8.80 & 0.17 \\
C3a & C & 35.57 & 5.35 & 1.96 & 0.24 & 4.40 & 4.18 & 0.15 \\
& Bh & 30.98 & 7.07 & 1.36 & 3.80 & 14.08 & 7.70 & 0.38 \\
C3b & Ah & 31.70 & 2.52 & 2.88 & 1.32 & 7.48 & 5.72 & 0.29 \\
\hline & 67.28 & 22.36 & 1.26 & 1.12 & 9.68 & 12.32 & 0.32 \\
\hline
\end{tabular}

Tabla 3. Concentraciones de elementos asimilables en los perfiles. Concentration of available cation elements throughout the profiles. 
Pinsapares agradativos Pinsapares maduros

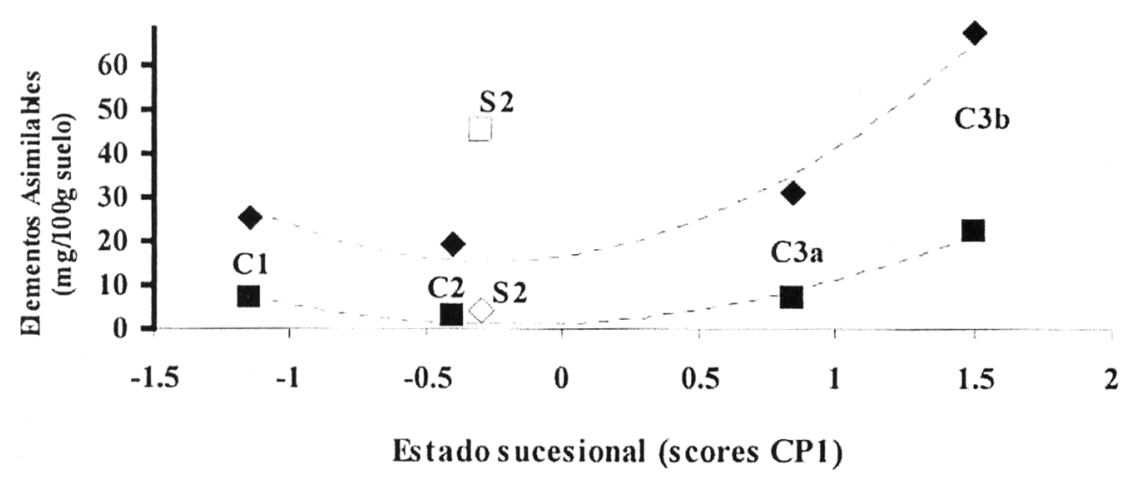

Figura 2. Concentración de $\mathrm{Ca}^{2+}$ (rombos) y $\mathrm{Mg}^{2+}$ (cuadrados) asimilables (extraible con DTPA) en el suelo superficial (horizonte Ah), y estado sucesional de la masa (coordenadas de cada parcela sobre el primer eje de un análisis de componentes principales con variables de la estructura del dosel forestal), en pinsapares calcícolas (símbolos sombreados; parcelas $\mathrm{C} 1, \mathrm{C} 2, \mathrm{C} 3$ a y C3b) y serpentinícolas (símbolos claros, parcela S2). DTPA-extractable Ca ${ }^{2+}$ (rhombus) y $\mathrm{Mg}^{2+}$ (squares) concentrations in surface soils (Ah horizon), and stand successional stage (plot scores on the first axis of a principal component analysis carried out with canopy structural variables), in calcareous (shaded symbols; Plots C1, C2, C3a and C3b) and serpentinic (open symbols, plot S2) pinsapo-fir forest.

la sucesión, las diferencias no fueron estadísticamente significativas (fig. 3 ). Incrementos sucesionales en la cantidad de hojarasca están ampliamente documentados, y se atribuyen al aumento de la biomasa total de la comunidad vegetal a lo largo de la sucesión y la consiguiente acumulación en el suelo de restos orgánicos (Vitousek \& Reiners, 1975; Hontoria et al., 1999; Entry \& Emmingham, 1994), a lo cual podría sumarse un efecto simultáneo de ralentización de la tasa de descomposición de la hojarasca (Hart \& Stark, 1997).

Las relaciones $\mathrm{C} / \mathrm{N}$ de la hojarasca, cercanas a 30 , no mostraron diferencias significativas a lo largo de la sucesión en pinsapares calcícolas, lo que sugiere un potencial de inmovilización neta del amonio durante su descomposición. La relación $\mathrm{C} / \mathrm{N}$ de la hojarasca es un índice del estado de humificación de la M.O. y permite predecir si el $N$ que contiene será, de forma neta, inmovilizado por los microorganismos edáficos que intervienen en los procesos de descomposición, o liberado como amonio al suelo (mineralización neta). En este último caso, el contenido de $\mathrm{N}$ de la hojarasca excede la demanda de $\mathrm{N}$ de los microorganismos descomponedores, y dicho exceso de la demanda queda como amonio disponible en el suelo para las plantas. Se ha demostrado que en hojarascas con $\mathrm{C} / \mathrm{N}>25$, durante su descomposición el $\mathrm{N}$ queda secuestrado por los microorganismos (Paul y Clark, 1989).

\subsection{Estado nutricional}

En la figura 4 aparecen las concentraciones de $\mathrm{N}$ y $\mathrm{P}$ total en acículas de los pinsapares de estudio, comparadas con los valores de estos macronutrientes en suelo superficial y hojarasca. Existe un incremento del contenido de $\mathrm{N}$ total en acículas $\left(17 \mathrm{mg} \cdot \mathrm{g}^{-1}\right.$ en pinsapares agradativos, $21 \mathrm{mg} \cdot \mathrm{g}^{-1}$ en pinsapares maduros; ANOVA, $\mathrm{P}<0.05$ ) paralelo al incremento de $\mathrm{Ni}$ $\left(\mathrm{N}-\mathrm{NH}_{4}{ }^{+}+\mathrm{N}-\mathrm{NO}_{3}{ }^{-}\right)$en el suelo y de $\mathrm{Nt}$ en la hojarasca, a lo largo de la sucesión (figura 4 


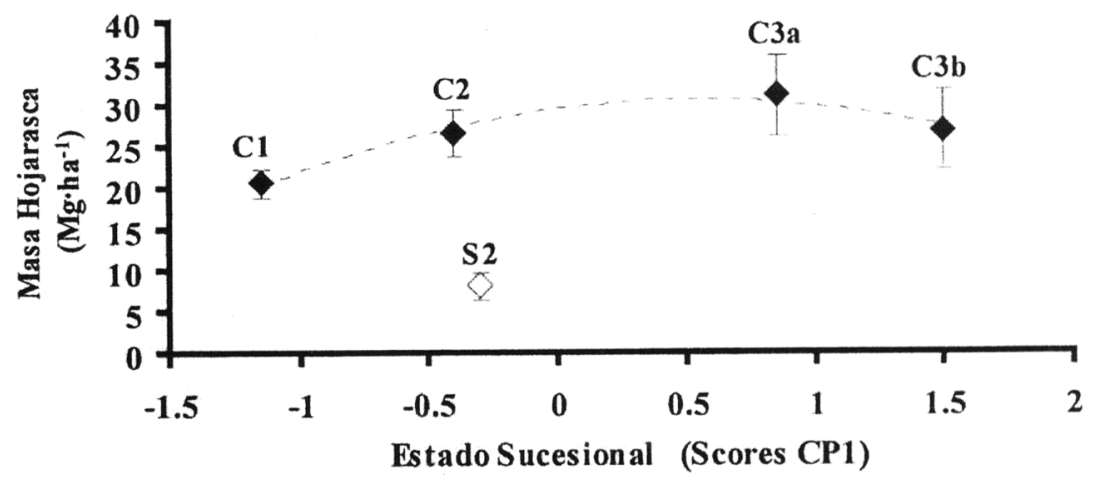

Figura 3. Cantidad de hojarasca (capas $\mathrm{L}+\mathrm{F}+\mathrm{H}$ ) por unidad de superficie en pinsapares calcícolas, incluyendo masas en fase agradativa (Sierra de las Nieves-Yunquera, parcelas C1 y C2) y en fase madura (Sierra de las Nieves-Ronda, parcelas C3a y C3b); y en un pinsapar serpentinícola en fase agradativa (Reales de Sierra Bermeja, parcela S2 cuya estructura del dosel es comparable al de la parcela C2). Litter mass ( $L+F+H$ layers) in an area basis, in calcareous, pinsapo-fir forests including stands in agradative (Sierra de las Nieves, plots $\mathrm{Cl}$ and C2) and old-growth (Sierra de las Nieves, Plots C3a and C3b) phases; and in a serpentinic, agradative, pinsapo-fir forest (Reales de Sierra Bermeja, plot 22 which canopy structure is comparable to that in the C2 plot).

A). En la parcela C3b, correspondiente al estadio sucesional más avanzado, los contenidos foliares de $\mathrm{N}$ y $\mathrm{P}$ tienden a descender, probablemente a causa de la senectud de los individuos.

Las concentraciones foliares de $\mathrm{N}$ en pinsapos calcícolas superan en líneas generales los umbrales críticos de deficiencia fijados en $11 \mathrm{mg} \cdot \mathrm{g}^{-1}$ para numerosas especies de pino (e.g. Romanyá \& Vallejo 1995), y entre 12 y $15 \mathrm{mg}$ $\mathrm{g}^{-1}$ para diversas especies de abeto (e.g. Binns et al. 1980). Para el abeto noruego, Vesterdal (1999) fijaba en $0.45 \mathrm{mg} \cdot \mathrm{g}^{-1}$ la concentración de P foliar crítica, por debajo de la cuál podía hablarse de sitios con suelos altamente deficitarios en P. Las concentraciones de P de los pinsapares calcáreos de estudio mantienen niveles bajos pero en ningún caso deficitarios (figura $4 \mathrm{~B}$ ).

En ocasiones los términos "estrés nutricional" y "deficiencias nutricionales" suelen considerarse sinónimos. Sin embargo, aunque una situación de estrés puede en efecto desencadenar desequilibrios fisiológicos atribuibles a deficiencias nutricionales, no se trata de términos equivalentes. Así, aunque los análisis foliares de los pinsapares calcáreos en fase de exclusión de tallos (fase del desarrollo forestal en la cual la competencia intraespecífica por nutrientes es más alta), mostraron concentraciones mínimas de $\mathrm{N}$ y $\mathrm{P}$, indicativas de un estrés nutricional general, no aparecen desequilibrios nutricionales ya que las relaciones $\mathrm{N} / \mathrm{P}$, en torno a 25 , pueden considerarse como normales.

En el ciclo del $\mathrm{N}$ tienen más importancia los procesos biológicos (fijación, mineralización, nitrificación, desnitrificación, etc.) mientras que en el del $\mathrm{P}$ son protagonistas los procesos geoquímicos (meteorización, adsorción sobre minerales secundarios y precipitación de fosfatos insolubles, etc.). Esto explica que la relación N/P aumente significativamente a lo largo de la sucesión forestal sobre calizas (desde promedios de 24.5 a 34.7 , ANOVA, $\mathrm{P}<0.05$ ), ya que la capitalización de materia orgánica implica, como hemos visto en los apartados anteriores, 

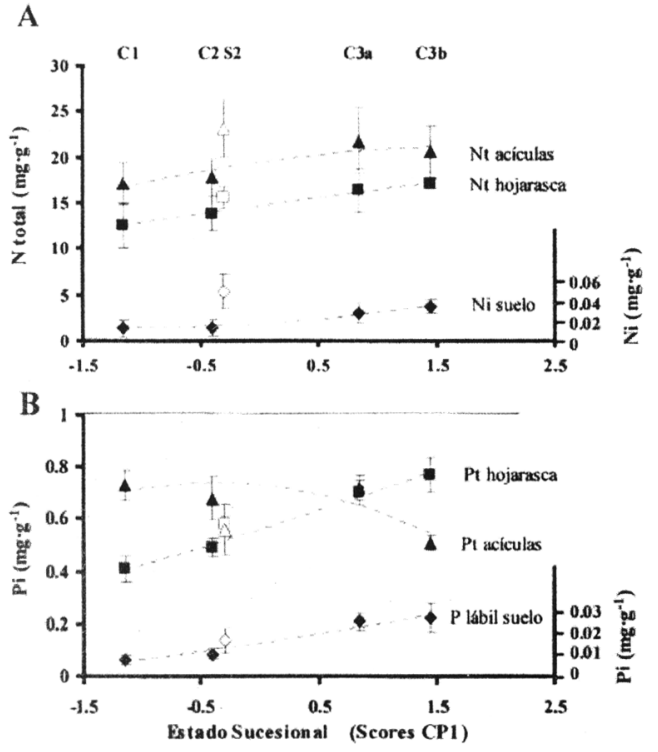

Figura 4. Concentraciones de nitrógeno inorgánico $\left(\mathrm{NH}_{4}^{+}+\mathrm{NO}_{2}^{-}\right)$extraíble con $\mathrm{KCl}$ en suelo superficial (horizonte Âh), y de nitrógeno total en hojarasca y acículas (A); y concentraciones de fósforo inorgánico lábil extraíble con $\mathrm{NaHCO}_{3}$ en suelo superficial (horizonte $\mathrm{Ah}$ ), y de fósforo total en hojarasca y acículas (B); en pinsapares calcícolas (símbolos sombreados; parcelas $\mathrm{C} 1, \mathrm{C} 2, \mathrm{C} 3 \mathrm{a}$ y C $3 \mathrm{~b}$ ) y serpentinícolas (símbolos claros, parcela S2). Concentrations of $\mathrm{KCl}$-extractable inorganic $\mathrm{N}$ $\left(\mathrm{NH}_{4}^{+}+\mathrm{NO}_{3}{ }^{-}\right)$in surface soils (Ah horizon), and total $N$ in litter and needles $(A)$; and concentrations of $\mathrm{NaHCO}_{3}$ extractable inorganic $\mathrm{P}$ in surface soils (Ah horizon), and total $P$ in litter and needles $(B)$; in calcareous (shaded symbols; Plots C1, C2, C3a and $C 3 b$ ) and serpentinic (open symbols, plot S2) pinsapo-fir forests.

incrementos en los contenidos de $\mathrm{N}$ que son proporcionalmente mayores que los de P. Por contra, al igual que ocurría en el suelo y la hojarasca, las relaciones $\mathrm{C} / \mathrm{N}$ de los tejidos vegetales no mostraron tendencias sucesionales estadísticamente significativas, indicando que la eficiencia de uso de $\mathrm{N}$ por los árboles es similar en los pinsapares jóvenes y maduros (Waring \& Schlesinger 1985) (fig. 1).
2. El pinsapar de Sierra Bermeja. La singularidad de los pinsapares serpentinícolas.

\subsection{Perfiles y suelo superficial}

El perfil de S2 ha sido clasificado como Hapludoll oxiácuico. Este suelo mostró tonos (hue) más rojizos que el resto de perfiles, como resultado de la alteración de las peridotitas. Esta roca, por un complejo proceso de meteorización, se hidrata dando lugar a suelos serpentinícolas de fina textura y abundantes óxidos de hierro. El horizonte superficial mostró valores de $\mathrm{pH}$ de entre 6.2 y 7.1 , frente a los valores próximos a 8 del conjunto de suelos calcáreos. El complejo de cambio está dominado por el calcio y el magnesio. Sobre serpentinas el porcentaje de la CEC aportado por el $\mathrm{Mg}^{2+}$ llega a superar el $50 \%$, mientras que en los suelos calcáreos sucesionalmente comparables está en torno al 18\% (tab. 2).

El estado sucesional de S2 es comparable a $\mathrm{C} 2$, sin embargo sus contenidos en C.O. y $\mathrm{N}$ están próximos a los de pinsapares maduros (fig. 1). Estos contenidos de materia orgánica presentes en S2 no pueden por tanto atribuirse a la estructura de su comunidad vegetal y probablemente sean debidos a mayores tasas de renovación de hojarasca y de humificación. En este sentido, a los dos factores formadores seleccionados como principal fuente de variación: roca madre y estado sucesional de la comunidad vegetal (como tiempo), deberíamos añadir, dentro del factor formador clima, la mayor pluviometría registrada en Sierra Bermeja (por encima de $1900 \mathrm{~mm}$ frente a los valores de la serranía de Ronda, en torno a $1100 \mathrm{~mm}$ ), como otra diferencia notable, capaz de justificar la existencia de un ciclado más activo de la hojarasca de S2 (Liétor, 2001).

Los altos valores de carbono orgánico y nitrógeno contrastan con las bajas relaciones $\mathrm{Ca}^{2+} / \mathrm{Mg}^{2+}$ y K$/ \mathrm{Mg}^{2+}$ que aparecen sobre esta litología tanto para los contenidos en elementos cambiables como para los asimilables (tabs. 2 y 3; fig. 2). Parte del carácter fitotóxico de los 
suelos desarrollados sobre serpentinas ha sido tradicionalmente atribuido a los niveles extraordinariamente altos de $\mathrm{Mg}$ asimilable (e.g. Kruckeberg, 1984; Proctor \& Nagy, 1992), responsables también de limitar la disponibilidad de $\mathrm{Ca}^{2+}$ y K+(Krám et al., 1997). Otra peculiaridad química destacable de S2 es la ausencia de carbonato cálcico equivalente, lo cual limita la capacidad tamponante y estructural $\left(\mathrm{el} \mathrm{Ca}^{2+}\right.$ es fundamental para la formación de agregados estables) de los suelos desarrollados sobre serpentinas.

Los valores de la relación $\mathrm{C} / \mathrm{N}$ del suelo no mostraron diferencias significativas entre pinsapares agradativos de distinta litología, aunque son algo menores sobre serpentinas. En todo caso, quedan dentro del intervalo promedio de C/N entre 15 y 20 para suelos de bosques templados (Paul y Clark, 1989). Los elevados niveles de $\mathrm{N}$ mineral de los suelos de los pinsapares serpentinícolas de Sierra Bermeja resultan impropios de suelos forestales desarrollados a partir de esta litología, más aún cuando se trata de ecosistemas mediterráneos, considerados típicamente como deficitarios en $\mathrm{N}$ mineral (Read \& Mitchell 1985). Así, los valores de S2 tanto para nitrógeno inorgánico $(\mathrm{Ni})\left(\mathrm{N}-\mathrm{NH}_{4}^{+}+\mathrm{N}-\mathrm{NO}_{3}{ }^{-}\right)$ como para nitrógeno total $(\mathrm{Nt})$ quedan fuera de la tendencia observada en las otras parcelas, siendo en todo caso superiores a los de la localidad sucesionalmente comparable (C2).

\subsection{Hojarasca}

La capa de hojarasca está más homogéneamente distribuida en pinsapares calcáreos (CV entre muestras alrededor del $50 \%$ en los pinsapares de Yunquera y Ronda, frente al casi $85 \%$ de Sierra Bermeja). En la figura 3 se aprecia que los pinsapares calcícolas agradativos presentan mayor cantidad de hojarasca por unidad de superficie que los pinsapares serpentinícolas del mismo estado sucesional (más de $20 \mathrm{Mgha}^{-1}$ frente a 12.8 $\mathrm{Mgha}^{-1}$ ) (ANOVA, $\left.\mathrm{p}<0.05\right)$. Esta observación concuerda con la hipótesis antes propuesta acerca de la existencia de mayores tasas de humificación en Sierra Bermeja (Liétor, 2001). La relación $\mathrm{C} / \mathrm{N}$ de la hojarasca es menor en los pinsapares serpentinícolas (ANOVA, $p<0.05$ ), integrándose dentro del rango en el que es posible mineralización neta (Paul y Clark, 1989).

\subsection{Estado nutricional}

Las acículas de pinsapares agradativos sobre serpentinas mostraron concentraciones medias de Nt significativamente superiores a las de los desarrollados sobre calizas (por encima de $23 \mathrm{mg} \cdot \mathrm{g}^{-1}$ en $\mathrm{S} 2$, y no superior a 18 $\mathrm{mg} \cdot \mathrm{g}^{-1}$ en $\mathrm{C} 2$; ANOVA, $\mathrm{P}<0.05$ ). Por el contrario, el $\mathrm{P}$ total es superior en las acículas de pinsapos calcícolas; ANOVA, $\mathrm{P}<0.05$. (fig. 4). Un incremento en el contenido foliar de $\mathrm{N}$ se puede considerar una consecuencia de altas concentraciones de $\mathrm{N}$ mineral en el suelo. En este sentido, incrementos de la concentración foliar de $\mathrm{N}$ paralelos a mayores disponibilidades edáficas de este elemento han sido observados en ecosistemas mediterráneos (i.e. Sabaté et al. 1995) y en abetales norteamericanos (Van den Driessche 1974). La concentración de N en acículas del pinsapar de Sierra Bermeja supera los niveles medios encontrados para diversas especies de coníferas no sometidas a entradas de nutrientes por deposición atmosférica (Aber et al. 1998), estando muy por encima de los niveles citados en el apartado 1.3.

La mayor cantidad de materia orgánica del suelo superficial de S2 aporta un capital de macronutrientes adecuado, quedando reflejado en la figura 1 el desplazamiento de esta parcela hacia el grupo de pinsapares maduros y en la figura $4 \mathrm{~A}$ el paralelismo existente entre los altos contenidos de $\mathrm{N}$ en suelo, hojarasca $\mathrm{y}$ acículas. Sin embargo, los contenidos foliares de P total son bajos (fig. 4 B). Los árboles de los pinsapares serpentinícolas poseen concentraciones de $\mathrm{P}$ muy próximas al valor crítico de $0.45 \mathrm{mg} \mathrm{g}^{-1}$ propuesto por Vesterdal 
(1999) y las relaciones N/P son significativamente superiores a las de sus homólogos sobre calizas (ANOVA, $\mathrm{P}<0.05$ ), indicando un claro desequilibrio nutricional $\mathrm{N}-\mathrm{P}$ en estos árboles.

En este sentido, es probable que la alta disponibilidad de $\mathrm{N}$ tenga como efecto indirecto una demanda adicional de $\mathrm{P}$ por parte de las plantas, que no se ve satisfecha por la tasa de suministro de P desde el suelo. De hecho, la roca madre de los suelos serpentinícolas es pobre en P. Ello determinaría que el P actúe como nutriente limitante en el pinsapar serpentinícola (Liétor 2001), cuando lo habitual es que sea el $\mathrm{N}$ el que juegue ese papel en ecosistemas forestales templados. Asimismo, bajas concentraciones de $\mathrm{P}$ foliar pueden ser producto del deterioro de las micorrizas y de las raíces finas debido al efecto nocivo del exceso de $\mathrm{N}$ mineral en el suelo (Gundersen 1998). La elevada disponibilidad de $N$ en los suelos de los pinsapares de Sierra Bermeja también justifica las diferencias significativas entre las relaciones $\mathrm{C} / \mathrm{N}$ de los tejidos vegetales, menores en estos bosques en relación con $\mathrm{C} 2$; ANOVA, $\mathrm{P}<0.05$.

Hemos señalado que, tradicionalmente, los suelos asentados sobre serpentinas se han considerado fitotóxicos, por sus elevados niveles de magnesio y metales pesados; y estériles, por la escasez de nutrientes esenciales en la roca madre, principalmente $\mathrm{N}, \mathrm{P}$ y $\mathrm{K}$ (Kruckeberg, 1984; Brooks, 1987). Sin embargo, en lo referente a formas de $\mathrm{N}$ en el suelo y la hojarasca, los pinsapares de Sierra Bermeja han mostrado valores altos, circunstancia que queda reflejada en los contenidos de $\mathrm{Nt}$ foliares de su vegetación (fig. 4 A). La existencia de entradas atmosféricas contaminantes de compuestos nitrogenados a estos pinsapares, descrita de forma preliminar por Liétor (2002), justificaría todos estos resultados. Dichas entradas pueden estar desencadenando un proceso de saturación por $\mathrm{N}$, cuya disponibilidad puede llegar a superar la capacidad de absorción y retención por parte de plantas, suelo y biomasa microbiana. Por otra parte un exceso de asimilación de $\mathrm{N}$ puede producir desajustes fisiológicos en los árboles. El mantenimiento a largo plazo, o la intensificación de estas entradas de $\mathrm{N}$, ocasionaría de forma paralela una progresiva acidificación, previsible habida cuenta de los ínfimos niveles de carbonato cálcico equivalente de las serpentinas. Esto acentuaría el lavado de cationes básicos, cuyos niveles son de por si bajos en los suelos desarrollados sobre peridotitas; disminuiría la disponibilidad de nutrientes esenciales como el P (Carreira et al. 1997) y el Mo, también deficientes en esta litología; e incrementaría la solubilidad de cationes acidificantes tóxicos, principalmente aluminio y metales pesados, cuyos niveles son de forma natural elevados en las serpentinas. Todos estos procesos pueden inducir declive generalizado en masas forestales (Aber 1998).

\section{CONCLUSIONES}

La capitalización de materia orgánica en el suelo a lo largo de la sucesión y la historia de manejo de cada localidad explican las diferencias encontradas en las características edáficas generales y en el grado de evolución de los suelos, en el pinsapar calcícola de la Sierra de las Nieves. Los contenidos en macronutrientes en el suelo siguen tendencias constantes de incremento a lo largo de la sucesión. La relación C/N permanece constante debido a que la capitalización de materia orgánica incrementa de manera proporcional las concentraciones de ambos elementos. Por el contrario, la relación N/P aumenta a lo largo de la sucesión por el mayor control geoquímico del ciclo del fósforo. En masas jóvenes (Yunquera) se ha detectado un estrés nutricional generalizado en los árboles, propio de la fase sucesional de exclusión de tallos, en la que la 
competencia intraespecífica es máxima; aunque no se manifiesta en desbalances nutricionales N/P. Dicho estrés nutricional se alivia en los pinsapares calcícolas maduros.

Las características peculiares de los suelos desarrollados sobre serpentinas son especialmente notables en las altas concentraciones de $\mathrm{Mg}^{2+}$ asimilable y las bajas relaciones $\mathrm{Ca}^{2+} / \mathrm{Mg}^{2+}$ y $\mathrm{K}^{+} / \mathrm{Mg}^{2+}$. La altas precipitaciones de Sierra Bermeja favorecen la humificación de la hojarasca, de forma que estos suelos presentan niveles de C.O. y $\mathrm{N}$ en el suelo superiores a los de pinsapares calcícolas sucesionalmente comparables. Aparecen en el pinsapar de Sierra Bermeja altas concentraciones de $\mathrm{N}$ mineral, impropias de suelos forestales mediterráneos, típicamente pobres en este nutriente, cuyo origen no puede relacionarse con su peculiar litología. Numerosos estudios en distintos tipos de ecosistemas forestales han relacionado elevadas disponibilidades de $\mathrm{N}$ en el suelo con la existencia de entradas crónicas atmosféricas de $\mathrm{N}$ (deposición de $\mathrm{N}$ ) lo que puede inducir síntomas de declive forestal (Síndrome de Saturación de Nitrógeno).

\section{BIBLIOGRAFÍA}

ABER, J., W. MCDOWELL, K. NADELHOFFER, A. MAGILL, G. BERNTSON, M. KAMAKEA, S. MCNULTY, W. CURRIE, L. RUSTAD \& I. FERNÁNDEZ -1998- Nitrogen saturation in temperate forest ecosystems. Hypotheses revisited. Bioscience 48(11): 921-934.

BENÍTEZ, I. -1984- Niveles de macro y microelementos en suelos forestales de la Serranía de Grazalema (Cádiz, España). Tesis de Licenciatura. Universidad de Córdoba.

BINNS, W.O., G.J. MAYHEAD \& J.M. MCKENZIE -1980- Nutrient defficiencies of conifers in British forests: An illustrated guide. Forestry Commission Leaflet 76, HMSO.

BROOKS, R.R. -1987-Serpentine and its vegetation. Croom Helm Ltd. Beckenham, UK (454pp).
Comisión de Métodos Analíticos del Instituto Nacional de Edafología "J.M. Albareda" -1984Congreso Nacional de Suelos.

CARREIRA J.A., J.R. ARÉVALO \& F.X. NIELL 1996- Soil degradation and nutrient availability in fire-prone Mediterranean shrublands of Southeastern Spain. Arid Soil Rechearch and Rehabilitation 10: 53-64.

CARREIRA, J.A., A.F. HARRISON, L.J. SHEPPARD \& C. WOODS -1997- Reduced soil $P$ availability in a Sitka spruce (Picea sitchensis Bong. Carr) plantation induced by applied acidmist: significance in forest decline. For. Ecol. Manag. 92: 153-166.

CORRAL, L. -1978- Estudio edafológico de la Sierra del Pinar, Grazalema (Cádiz). Tesis Doctoral. Universidad de Córdoba.

CORRAL, L., N. BELLINFANTE y G. PANEQUE -1980- Estudio edafológico de la Sierra del Pinar, Grazalema (Cádiz). I a VII. Anal. Edaf. Agrob. XXXIX.

DEL TORO, M., L. CORRAL y J. GIL -1996Evaluación de la aptitud de los suelos del Parque Natural "Sierra de Grazalema" para uso de pinsapar. Boletín de la Sociedad Española de la Ciencia del Suelo 3-2: 499-505.

ENTRY, J.A. \& W. H. EMMINGHAM - 1994 Influence of forest age on nutrient availability and storage in coniferous soils of the Oregon Coastal Range. Can. J. For. Res. 25: 114-120.

FAO -1977- Guía para la descripción de perfiles de suelo. 2nd Ed. Roma.

GONZÁLEZ, J.L. -1983- Estudio de la materia mineral de suelos de la Serranía de Grazalema. Tesis Doctoral. Universidad de Sevilla.

GRUBB, P.J. -1998- A reassessment of the strategies of plants which cope with shortages of resources. Perspectives. Plant Ecology, Evolution and Systematics. Vol 1/1: pp 3-31.

GUNDERSEN, P., B.A. EMMETT, O.J. KJØNASS, C.J. KOOPAMANS \& A. TIETEMA -1998Impact of nitrogen deposition on nitrogen cycling in forests: a synthesis of NITREX data. For. Ecol. Manag. 101:37-55.

HART, S.C. \& J.M. STARK -1997- Nitrogen limitation of the microbial biomass in an oldgrowth forest soil. Ecoscience 4 (1): 91-98.

HERRICK, J.E. -1995-Simple method for determination of mass loss rates for soilcontaminated samples in decomposition studies. 
Pedobiologia 39: 74-77.

HONTORIA, C., J.C. RODRÍGUEZ \& A. SAÁ 1999-Relationships between soil organic carbon and site characteristics in Peninsular Spain. Soil Sci. Soc. Am. J. 63: 614-621.

JOHN, M.K. -1970- Colorimetric determination of phosphorus in soil and plant materials with ascorbic acid. Soil Science 109(4): 214-220.

KEENEY, D.R. \& D.W. NELSON -1982- Nitrogen. Inorganic forms. En: Page, A.L., R.H. Miller y D.R. Keeney (eds). Methods of Soil Analysis. Part 2. Chemical and microbiological properties (pp 643-699). Book series $n^{\circ} 9$ Soil Science Society of America, Madison.

KILLHAM, K. -1994- The ecology of soil nutrient cycling. En: Killham, K.(ed). Soil Ecology (pp 89-149). Cambridge University Press, Cambridge .

KRÁM, P., K. HRUSKA, B.S. WENNER, C.T. DRISCOLL \& C.E. JOHNSON -1997- The biogeochemistry of basic cations in two forested catchments with contrasting lithology in the Czech Republic. Biogeochemistry 37: 173-202.

KRUCKEBERG, A.R. -1984- California serpentines: Flora, vegetation, geology, soils and management problems. University of California Press. Los Angeles, USA (180pp).

LIÉTOR, J. -2001- Patrones de disponibilidad y limitación por nutrientes como indicadores de estado en masas de pinsapar (Abies pinsapo Boiss.). Tesis Doctoral. Universidad de Jaén.

LIÉTOR, J., R. GARCÍA-RUIZ, B. VIÑEGLA, V. OCHOA, J.C. LINARES, B. HINOJOSA, M.T. SALIDO y J.A. CARREIRA -2002-Variabilidad Biogeoquímica en masas de pinsapar: efecto de la litología y el estado sucesional. Ecología 16: 45-57.

LINDSAY, W.L. \& W.L. NOVELL -1978Development of DTPA soil test for zinc, iron, manganese and copper. Soil Sci. Soc. Am. J. 42: 421-428.

MÉRIDA, J. -1983- Estudio de la materia orgánica de suelos de la Serranía de Grazalema. Tesis Doctoral. Universidad de Sevilla.

MUIR, J.W. -1969- A natural system of soil classification. Jcurnal of Soil Science. Vol 20, $\mathrm{N}^{\circ} 1: 153-166$.

Munsell Color Company Inc. -1990- Munsell Soil Color Charts. Kollmorgan Instruments Corporation. New York, USA.
PANEQUE, G. y L. CORRAL -1982- Propiedades químicas de suelos y niveles de nutrientes en acículas de Abies pinsapo de Grazalema (Cádiz, España). Anales de Edafología y Agrobiología 41: 197-209.

PARKINSON, J.A. \& S.E. ALLEN -1975- A wet oxidation procedure suitable for the determination of nitrogen and mineral nutrients in biological materials. Commun. Soil Plant Anal 6: 1-11.

PAUL, E.A. \& F.E. CLARK -1989-Soil Microbiology and Biochemistry. Academic Press. San Diego, California (USA). 267pp.

PÉREZ LA TORRE, A.V., D. NAVAS, Y. GIL y B. CABEZUDO -1998- Datos sobre la flora y vegetación de la Serranía de Ronda (Málaga, España). Acta Bot. Malacitana 23:149-191.

PROCTOR, J. \& L. NAGY -1992- Ultramafic rocks and their vegetation: an overview. En: Baker, A.M.J., J. Proctor y R.D. Reeves (eds). The vegetation of ultramafic (serpentine) soils (pp 469-494).

RANGER, I., R. MARQUES \& M. COLINBELGRAND - 1997- Nutrient dynamics during the development of a Douglas fir (Pseudotsuga menziesii Mirb.) stand. Acta Oecologica 18(2): 73-90.

READ, D.J. \& D.T. MITCHELL -1985Decomposition and mineralization processes in Mediterranean-type ecosystems and in heathlands of similar structure. En: Kruger, F.J., D.T. Mitchell \& J.U.M. Jarvis (eds). Mediterranean-Type Ecosystems. The role of nutrients (pp 208-232). Ecological Studie 43. Springer-Verlag.

ROBERT, B., G. BERTONO, D. SAYAG \& P. MASSON -1996-Assessment of mineral nutrition of cork oak through foliar analysis. Commun. Soil Sci. Plant Anal. 27(9-10): 20912109.

ROMANYA, J. \& R. VALLEJO -1995- Nutritional status and deficiency diagnosis of Pinus radiata plantations in Spain. Forest Science 42: 192197.

RUIZ, J. -1994- Los pinsapares andaluces. En: Asociación Forestal Andaluza (ed). Gestión y Conservación de los Pinsapares Andaluces (pp11-27).

SABATÉ, S., A. SALA \& C.A. GRACIA -1995Nutrient content in Quercus ilex canopies: 
Seasonal and spatial distribution within a catchment. Plant and Soil 168-169: 297-304.

Soil Conservation Service -1972- Soil survey laboratory methods and procedures for collecting soil samples. Soil Survey Investigations Report, 1.

Soil Survey Staff - 1993- Soil Survey Manual. USDA. Soil Survey Staff -1999- Keys to Soil Taxonomy, 8th Ed. USDA.

VAN DEN DRIESSCHE, R. -1974- Prediction of mineral nutrient status of trees by foliar analysis. The Botanical Review 40(3): 347-394.

VAN MIEGROET, H., D.W. JOHNSON \& D.W. COLE -1990- Soil nitrification as affected by $\mathrm{N}$ fertility and changes in forest floor $\mathrm{C} / \mathrm{N}$ ratio in four forest soils. Can. J. For. Res. 20: 10121019.

VESTERDAL, L. -1999- Influence of soil type on mass loss and nutrient release from decomposing foliage litter of beech and Norway spruce. Can. J. For. Res. 29(1): 95-105.

VITOUSEK, P.M. \& W.A. REINERS -1975Ecosystem, succession and nutrient retention: a hypothesis. BioScience 25: 376-381.
WARING, R.H. \& W.H. SCHLESINGER -1985Forest Ecosystems. Concepts and Management. Academic Press, London.

YUSTA, A. -1984- Geoquímica de las alteraciones superficiales de peridotitas de Los Reales (Málaga). Tesis Doctoral. Universidad de Granada.

Aceptado para su publicación en octubre de 2003

Dirección de los autores. J. A. CARREIRA, Roberto GARCÍA RUIZ y José LIÉTOR: Dpto. Biología Animal, Vegetal y Ecología, Ed. B-3, Paraje Las Lagunillas s/n 23071, Universidad de Jaén, Jaén; J. C. LINARES: Dpto. Ciencias Ambientales, Ctra. Utrera, km. 1 - 41013, Universidad Pablo de Olavide, Sevilla; J. M. MARTín GARCÍA: Dpto. Geología, Ed. B-3, Paraje Las Lagunillas s/n 23071, Universidad de Jaén, Jaén. 